\title{
RANDOM WALKS ON ABELIAN GROUPS
}

\author{
R. M. DUDLEY ${ }^{1}$
}

The purpose of this note is to prove the following

Theorem. A countable abelian group $G$ has a recurrent random walk (reaching each element of the group) just in case its rank (maximal number of linearly independent elements) is at most 2.

By random walk I shall mean a Markov chain with homogeneous transition probabilities $p(a, b)=p(0, b-a)=p(b-a)$ and nonnegative integral times. Temporal homogeneity is also assumed, and usually the initial position will be fixed at 0 (the identity of $G$ ). Letting $x(n)$ be a typical sample function, the walk is recurrent just in case for each $g \in G, \operatorname{Pr}(x(n)=g$ for some $n)=1$. This will hold just in case $\sum_{n} p_{n}(g)$ diverges (see $[1, \mathrm{p} .1]$ ) where $p_{1}=p$ and for each $n \geqq 1, p_{n+1}(g)=\sum_{h \in G} p(h) p_{n}(g-h)$. A walk will be called symmetric if $p(-g)=p(g)$ for each $g \in G$.

The following two lemmas are presumably well known; the proof of the second was kindly suggested by Professer F. Spitzer.

LEMMA 1. If the (discrete, abelian) group $G$ has a recurrent random walk, so does any subgroup $H$ of $G$.

PROOF. Clearly if $G$ has a recurrent walk it has one starting at 0 . Let $x(n, \omega)$ be such a walk where $\omega$ belongs to an underlying probability space $\Omega$. Let $m(\omega)$ be the least $m$ such that $x(m, \omega) \in H(m$ is defined for almost all $\omega)$. Set $q(h)=\operatorname{Pr}(x(m(\omega), \omega)=h)$ for each $h \in H$. Clearly $q$ defines a probability distribution on $h$, and the random walk with transition probability $q$ starting at 0 is recurrent since the original walk eventually hits each point of $H$ with probability one and the new walk is more likely to have hit an $h \in H$ by time $n$ than the old was.

Lemma 2. Let $z(k, \omega)$ be a symmetric rändom walk on $Z \oplus Z$ (where $Z$ is the additive group of integers) with $p(a)=0$ except for finitely many $a \neq(0,0)$ and positive probability of eventually hitting any fixed element. Then $z(k)$ is recurrent.

Proof. Let $p\left(\left(m_{j}, n_{j}\right)\right)=\alpha_{j}, j=1, \cdots, N$, where $\sum_{j=1}^{N} 2 \alpha_{j}=1$ and $\left(m_{j}, n_{j}\right) \neq\left(-m_{k},-n_{k}\right)$ for all $j$ and $k$. Then

Received by the editors March 27, 1961.

${ }^{1}$ National Science Foundation Fellow. 


$$
\begin{aligned}
\sum_{k=0}^{\infty} \operatorname{Pr}(z(k) & =0)=\sum_{k=0}^{\infty} \int_{0}^{1} \int_{0}^{1}\left[\sum_{j=1}^{N} 2 \alpha_{j} \cos \left(2 \pi\left(m_{j} x+n_{j} y\right)\right)\right]^{k} d x d y \\
& =\lim _{k \rightarrow \infty} \int_{0}^{1} \int_{0}^{1} \frac{1-\left[\sum_{j=1}^{N} 2 \alpha_{j} \cos \left(2 \pi\left(m_{j} x+n_{j} y\right)\right)\right]^{k+1}}{1-\sum_{j=1}^{N} 2 \alpha_{j} \cos \left(2 \pi\left(m_{j} x+n_{j} y\right)\right)} d x d y .
\end{aligned}
$$

For odd $k \rightarrow \infty$ the sequence of integrands is monotone increasing: hence by the monotone convergence theorem, the limit is

$$
\begin{aligned}
& \int_{0}^{1} \int_{0}^{1} \frac{1}{1-\sum_{j=1}^{N} 2 \alpha_{j} \cos \left(2 \pi\left(m_{j} x+n_{j} y\right)\right)} d x d y \\
& \quad=\int_{0}^{1} \int_{0}^{1} \frac{d x d y}{\alpha x^{2}+\beta x y+\gamma y^{2}+o\left(x^{2}+y^{2}\right)}=+\infty .
\end{aligned}
$$

Thus there is probability one of returning to 0 , hence probability one of eventually hitting any element, q.e.d.

Proof of the THEOREM. Lemma 1 implies, using the known fact that there is no recurrent walk on $Z \oplus Z \oplus Z$ (see [1, p. 3]) that the rank of a discrete abelian group with a recurrent walk is at most 2 .

The converse, of course, is harder to prove. The idea of the proof is to construct a sequence of recurrent walks on finitely generated subgroups which converge so rapidly to a walk on the whole group that it is also recurrent. We may assume that $G$, our abelian group of countable cardinality and rank at most 2 , is not finitely generated, for if it is, the existence of a recurrent walk follows almost immediately from Lemma 2. If $G$ is of rank 1 , let $a_{1}$ be any element of infinite order: if the rank is 2 , let $a_{1}$ and $a_{2}$ be any two linearly independent elements. Choose (further) $a_{i}$ such that $a_{1}, a_{2}, \ldots$ generate $G$ and for each $n, a_{n+1}$ is not in the subgroup $G_{n}$ generated by $a_{1}, \cdots, a_{n}$.

We define inductively a sequence $\left\{p^{(n)}\right\}$ of symmetric transition probabilities with $p^{(n)}$ concentrated in $G_{n}$ for each $n$. Let $p^{(1)}\left(a_{1}\right)$ $=p^{(1)}\left(-a_{1}\right)=1 / 2$ (unless $a_{1}$ is of order 2 , then $p^{(1)}\left(a_{1}\right)=1$ ) and $p^{(2)}\left(a_{1}\right)=p^{(2)}\left(-a_{1}\right)=p^{(2)}\left(a_{2}\right)=p^{(2)}\left(-a_{2}\right)=1 / 4$, unless $a_{1}$ and $/$ or $a_{2}$ is of order 2 in which case $p^{(2)}\left(a_{1}\right)$ and/or $p^{(2)}\left(a_{2}\right)$ is $1 / 2$. We want $p^{(n)}$ to define a recurrent walk on $G_{n}$ for each $n$; this is clearly true so far (it follows from Lemma 2 that any symmetric walk on an abelian group with two generators is recurrent).

Given $p^{(n)}$ defining a symmetric recurrent walk on $G_{n}$, we shall 
set $p^{(n+1)}(g)=\left(1-q_{n+1}\right) p^{(n)}(g)$ if $g \in G_{n}, p^{(n+1)}\left(a_{n+1}\right)=p^{(n+1)}\left(-a_{n+1}\right)$ $=q_{n+1} / 2\left(q_{n+1}\right.$ if $\left.2 a_{n+1}=0\right)$ and $p^{(n+1)}(g)=0$ otherwise, where $q_{n+1}$ is a sufficiently small positive number, to be chosen later.

By the fundamental theorem on finitely generated abelian groups, $G_{n}$ is always of the form $Z \oplus Z \oplus H$ or $Z \oplus H$ or $H$ where $H$ is a finite group. Since $p^{(n)}$ is symmetric and concentrated in finitely many points, the walk it defines returns with probability one into the finite group $H$, by Lemma 2 ; since there is positive probability of getting to each point of $H$ there is probability one of returning to 0 , and hence $p^{(n)}$ defines a recurrent walk on $G_{n}$.

Now to pick the $q_{n}$. Let $x^{(n)}(k, \omega)$ be the walk starting at 0 on $G_{n}$ defined by $p^{(n)}$. We know that $\sum_{k=1}^{\infty} \operatorname{Pr}\left(x^{(n)}(k, \omega)=0\right)$ diverges for each $n$. Choose $N_{1}$ so that

$$
\sum_{k=1}^{N_{1}} \operatorname{Pr}\left(x^{(1)}(k, \omega)=0\right)>1,
$$

and choose $Q_{11}, Q_{12}, \cdots>0$ so that

$$
\prod_{j=1}^{\infty}\left(1-Q_{1 j}\right)^{N_{1}} \sum_{k=1}^{N_{1}} \operatorname{Pr}\left(x^{(1)}(k, \omega)=0\right)>1 .
$$

Given $N_{1}, \cdots, N_{n-1}, Q_{11}, \cdots, Q_{n-1,1}, \cdots$ choose $N_{n}$ so that

$$
\sum_{k=N_{n-1}+1}^{N_{n}} \operatorname{Pr}\left(x^{(n-1)}(k, \omega)=0\right)>1
$$

and $Q_{n 1}, Q_{n 2}, \cdots$ positive and such that

$$
\prod_{j=1}^{\infty}\left(1-Q_{n j}\right)^{N_{n}} \sum_{k=N_{n-1}+1}^{N_{n}} \operatorname{Pr}\left(x^{(n-1)}(k, \omega)=0\right)>1 .
$$

Set $q_{n}=\min \left(Q_{1 n}, \cdots, Q_{n n}, r_{n}\right)$ where $r_{n}$ are any positive numbers less than 1 with $\prod_{n=1}^{\infty}\left(1-r_{n}\right)$ convergent. Finally, define

$$
p(g)=\prod_{n=m+1}^{\infty}\left(1-q_{n}\right) p^{(m)}(g) \quad \text { for } g \in G_{m} ;
$$

this is consistent, and $p$ is defined on all of $G$ since $G$ is the union of the $G_{n}$. For each $n$ we have

$$
\prod_{k=n}^{\infty}\left(1-r_{k}\right)=\prod_{k=n}^{\infty}\left(1-r_{k}\right) \sum_{g \in G_{n}} p^{(n)}(g) \leqq \sum_{g \in G_{n}} p(g)<1 .
$$

Thus $\sum_{g \in G} p(g)=1$, and $p$ can be used as a transition probability. Let $x(k, \omega)$ be a random walk starting at 0 with $p$ as transition function. Then for any positive integer $K$, 


$$
\operatorname{Pr}\left(x(k, \omega) \in G_{n}, k=0,1, \cdots, K\right)=\prod_{k=n+1}^{\infty}\left(1-q_{k}\right)^{K} .
$$

The conditional probability of being back at 0 after $j$ steps, given that we have not left $G_{n}$, is precisely $\operatorname{Pr}\left(x^{(n)}(j, \omega)=0\right)$. Thus

$$
\begin{aligned}
\sum_{j=N_{n-1}+1}^{N_{n}} \operatorname{Pr}(x(j, \omega)=0) & \geqq \sum_{j=N_{n-1}+1}^{N_{n}} \prod_{k=n+1}^{\infty}\left(1-q_{k}\right)^{j} \operatorname{Pr}\left(x^{(n)}(j, \omega)=0\right) \\
& \geqq \prod_{k=n+1}^{\infty}\left(1-q_{k}\right)^{N_{n}} \sum_{j=N_{n-1}+1}^{N_{n}} \operatorname{Pr}\left(x^{(n)}(j, \omega)=0\right) \\
& \geqq \prod_{k=n+1}^{\infty}\left(1-Q_{n k}\right)^{N_{n}} \sum_{j=N_{n-1}+1}^{N_{n}} \operatorname{Pr}\left(x^{(n)}(j, \omega)=0\right) \\
& \geqq 1 \text { by choice of the } Q_{n k} .
\end{aligned}
$$

Thus $\sum_{j=1}^{\infty} \operatorname{Pr}(x(j, \omega)=0)$ diverges. Since clearly there is positive probability of hitting each element, $x(n, \omega)$ is recurrent, q.e.d.

The largest discrete abelian group having a recurrent random walk seems to be the (restricted) direct sum of two copies of the additive group of rationals and countably many copies of the additive group of rationals mod 1. Calling a walk on a topological group recurrent when there is probability one of eventually hitting any open set, we see that a topological abelian group has a recurrent random walk if it has a dense subgroup of rank at most 2. Any compact abelian group has the recurrent random walk whose one-step transition probability is given by Haar measure. I hope to treat more general topological groups in a later paper.

The problem solved by our theorem was suggested by Professor Spitzer: I would like to thank him as well as Professor H. F. Trotter with whom I had several helpful discussions.

\section{BIBLIOGRAPHY}

1. K. L. Chung and W. H. J. Fuchs, On the distribution of values of sums of random variables, Four papers on probability, Memoirs Amer. Math. Soc. No. 6 (1951) 1-12.

Princeton University 